\title{
Modelling parasite density in ruminants
}

\author{
Steven I. Barry ${ }^{1} \quad$ Simon Brown ${ }^{2}$
}

(Received 4 August 2008; revised 5 January 2009)

\begin{abstract}
Models of parasite density in ruminants usually involve three nonlinear, coupled, ordinary differential equations for larval density, adult parasite density and host resistance. We deal with the inverse problem of objectively determining the nonlinear coefficients within the equations using available data. Experiments are suggested to allow efficient calculation of these coefficients. An alternative model is also proposed which reproduces key features of experimental results and shows the importance of including time delay equations in parasite modelling.
\end{abstract}

\section{Contents}

1 Introduction

C697

2 Solution method

C699

3 Testing

C700

http://anziamj . austms . org . au/ojs/index.php/ANZIAMJ/article/view/1397 gives this article, (C) Austral. Mathematical Soc. 2009. Published January 21, 2009. ISSN 1446-8735. (Print two pages per sheet of paper.) 


\section{Introduction}

Most ruminants, such as cattle and sheep, are infected by nematode parasites. Farmed ruminants tend to be kept at relatively high density and, despite stock rotation, the development of resistance to the parasite and chemical treatment, the parasite population density can be considerable. Wild populations tend to graze at lower density and range over a much larger area, so parasite population density is manageable. Understanding the parasite population dynamics leads to improvements in animal health and farm productivity.

Nematode parasites of ruminants have both a free living stage on pasture and a parasitic stage in the host. The larvae are ingested by the host where they develop into adults. The adults lay eggs that are deposited onto the pasture with the faeces in which the larvae develop. The parasites elicit an immune response from the host that affects parasite establishment, viability and parasite fertility. Roberts and Grenfell [6] proposed a model describing the larval population, L (number per unit area), the population of adults, A (number per unit area), and a generic immune response of the host, R:

$$
\begin{aligned}
\frac{d L}{d t} & =-(\beta+\rho) L+X_{1}(R) A \\
\frac{d A}{d t} & =\beta X_{2}(R) L-X_{3}(R) A \\
\frac{d R}{d t} & =\beta L-\sigma R .
\end{aligned}
$$


The rate at which larvae disappear from the pasture is determined by the rate at which they are ingested by the host, $\beta$, or die while on the pasture, $\rho$, and they appear at a rate, $X_{1}(R)$, that is often modelled as the product of the fertility of the adult parasites, $\lambda$, and the probability that an egg will develop into a larva, $q(R)$. The rate at which adults appear in the host depends on the probability that an ingested larva becomes established in the host, $X_{2}(R)$, and adults die in the host, $X_{3}(R)$. Host resistance to parasites increases as more larvae are ingested [1], $R^{\prime}=\beta L$, but decays with time if the host is not exposed to larvae, $R^{\prime}=-\sigma R$. Note that some models of parasite populations incorporate a stocking rate, $\mathrm{H}$, and use a density as number of larvae or adults per host [5]. However, since we do not study the effect of stocking rate, we analyse here the original formulation of the model by Roberts and Grenfell [6].

The effect of the host immune response on parasite establishment, $X_{2}$, fertility, $X_{1}$, and viability, $X_{3}$, is modelled by unknown functions that depend on R. While the model (1)-(3) captures many of the features of the dynamics of the biology, various arbitrary expressions have been used to model these coefficients depending on R. For example, some authors assumed $X_{2}$ to be constant [6], whereas others employed an exponential function of $\mathbf{R}[4,5]$. Similarly, $X_{3}$ has been taken to be constant $[4,6]$ or a 'sigmoidal' function of $\mathrm{R}$ [5]. It is expected that different parasites give rise to different functions $\mathrm{X}_{i}$; however, one generic model with variable parameters also models a wide variety of parasites. The aim of this article is to find the functions $X_{i}$ without a priori assuming a particular mathematical form.

Here we address the inverse problem of determining the form of these functions $X_{i}$, from the available experimental data. It is beyond the scope of this article to analyse experimental data in any depth. Our goal is to demonstrate techniques that can be used to find these coefficients, and hence guide experimentalists to what data are required and provide a method to test the viability of this model. 


\section{Solution method}

The goal here is to estimate $X_{1}(R), X_{2}(R)$ and $X_{3}(R)$ from experimental data $A(t)$ and $L(t)$. The values of $\beta, \rho$ and $\sigma$ are not known, but could be estimated indirectly from a range of experimental data $[4,5]$ or directly by nonlinear regression. In general, $R(t)$ is not known; however, one can estimate it from Equation (3).

In many experiments, parasite-naive animals are introduced to an area contaminated with larvae, hence $R(0)=A(0)=0$ while $L(0)>0$. Since $\sigma$ is small $[4,5,6]$ Equation (3) gives

$$
\mathrm{R}(\mathrm{t}) \approx \beta \int_{0}^{\mathrm{t}} \mathrm{L}(\tau) \mathrm{d} \tau
$$

with $R(t)$ monotically increasing. Whilst it is not necessary to make $\sigma=0$ to find $R$, as it can be found exactly from Equation (3), $\sigma$ is indeed small enough to be ignored over the time scales of one year studied here. The crucial aspect in calculating $R(t)$ is that it is monotonic for small $\sigma$ over the experimental period.

A single set of experimental data is insufficient to determine all the $X_{i}$ functions accurately. For example, for one experiment Equation (2) will have known terms $\frac{d A}{d t}, A(t), \beta$ and $L(t)$ and unknowns $X_{2}(R(t))$ and $X_{3}(R(t))$. This is ill-posed since, for example, setting $X_{3}=0$ still gives a mathematically feasible solution for $X_{2}$, albeit an incorrect one. The system is still ill-posed if the behaviour of the $X_{i}$ is restricted to physically realistic forms. Hence, two experiments are required to find an acceptable solution.

We assume two separate experiments, both with regions of monotonic $R(t)$, resulting in known data $L_{1}\left(t_{1}\right), A_{1}\left(t_{1}\right), L_{2}\left(t_{2}\right)$ and $A_{2}\left(t_{2}\right)$ at different times $t_{1}$ and $t_{2}$. That is, two different experiments are to be compared, with parasite variables monitored in both. These are then compared at times where the value of $R(t)$ was the same in both experiments. With $\sigma \approx 0$, then Equation (4) estimates $R$ with reasonable accuracy to give $R_{1}\left(t_{1}\right)$ and $R_{2}\left(t_{2}\right)$. 
Equation (2) is then written as

$$
\begin{aligned}
& \left.\frac{d A_{1}}{d t}\right|_{t=t_{1}}=X_{2}\left(R_{1}\left(t_{1}\right)\right) \beta L_{1}\left(t_{1}\right)-X_{3}\left(R_{1}\left(t_{1}\right)\right) A_{1}\left(t_{1}\right) \\
& \left.\frac{d A_{2}}{d t}\right|_{t=t_{2}}=X_{2}\left(R_{2}\left(t_{2}\right)\right) \beta L_{2}\left(t_{2}\right)-X_{3}\left(R_{2}\left(t_{2}\right)\right) A_{2}\left(t_{2}\right) .
\end{aligned}
$$

The system is simplified by choosing $t_{1}$ and $t_{2}$ such that $R_{1}\left(t_{1}\right)=R_{2}\left(t_{2}\right)=R$ giving two equations in two unknowns $X_{2}(R)$ and $X_{3}(R)$

$$
\left[\begin{array}{l}
X_{2}(R) \\
X_{3}(R)
\end{array}\right]=\left[\begin{array}{ll}
\beta L_{1}\left(t_{1}\right) & -A_{1}\left(t_{1}\right) \\
\beta L_{2}\left(t_{2}\right) & -A_{2}\left(t_{2}\right)
\end{array}\right]^{-1}\left[\begin{array}{l}
\left.\frac{d A_{1}}{d t}\right|_{t=t_{1}} \\
\left.\frac{d A_{2}}{d t}\right|_{t=t_{2}}
\end{array}\right] .
$$

This solution is repeated for each value of $R$ to generate the functional form for $X_{2}(R)$ and $X_{3}(R)$. The same process repeated on Equation (1) finds $\rho$ and $X_{1}(t)$.

Thus we are not attempting to design experiments which give the same $R(t)$ values. Instead we are exploiting the monotonic increase of $R(t)$ to be able to numerically choose times in both experiments where $R_{1}\left(t_{1}\right)=R_{2}\left(t_{2}\right)$. In doing so the equations are simplified and the coefficients found for this one value of $R$. The derivative $d A / d t$ is calculated by first fitting a regression curve through the $A(t)$ data. Once this smooth curve is obtained, differentiation is done numerically by simple central differencing or by analytic differentiation of the functional form.

\section{$3 \quad$ Testing}

This method was tested using a numerical experiment by assuming known functional forms $[5,6]$ for $\mathrm{X}_{i}$

$$
X_{1}=13797 \exp \left(-R \times 10^{-6}\right),
$$




$$
\begin{aligned}
& X_{2}=0.1278 \exp \left(-7.5 \times 10^{-6} R\right) \\
& X_{3}=12+13[1-\exp (-R / 551090)] .
\end{aligned}
$$

These functional forms were used to generate numerical experimental results for $L(t), A(t)$ and $R(t)$ under the two different experimental conditions of $A(0)=0$ and $\mathrm{L}(0)>0$, or $A(0)>0$ and $\mathrm{L}(0)=0$. The MATLAB differential equation solver, dsolve, was used and results checked against a separate Euler time step method, hence giving us confidence in the numerical accuracy of the result. The Euler time step was additionally checked for appropriate convergence and $\mathrm{O}(\mathrm{dt})$ accuracy.

Using these numerically generated results our goal was to use Equation (7) to re-generate $X_{i}(R)$, comparing these with the original assumed forms.

Figure 1 shows the scaled solutions $L(t), A(t)$ and $R(t)$ for both numerical experiments. The first uses an initial condition $\mathrm{L}(0)=40000$ larvae per unit area, $A(0)=R(0)=0$ and corresponds to an experiment in which parasitenaive animals graze contaminated pasture. The second uses $\mathrm{L}(0)=0$ and $A(0)=4000$ adults per unit area, and $R(0)=0$, perhaps corresponding to a situation in which animals are housed over a long winter during which the larvae on the pasture die yet sufficient adult parasites survive [8].

The solutions are scaled for clarity by the relative maxima of the functions: 4698312, 4148 and 619852 for L, A and R, respectively, in the left panel; and by 6774139,6462 and 772057 in the right panel. For these simulations $\rho=7, \sigma=0.01$ and $\beta=0.365[6]$.

Figure 2 shows the three functions $X_{1}(R), X_{2}(R)$ and $X_{3}(R)$ in the original form from Equations (8)-(10), scaled by 13797, 0.12775 and 21.7973 respectively, and the regenerated forms using the procedure above at 20 values of $R$. Also shown is the constant $\rho$ in both the original estimate of $\rho=7$ and the regenerated value. This has been scaled by 14 for convenience. The results indicate a good fit with the assumed forms for $X_{i}(R)$, although care must be taken near $R=0$ since the inversion in Equation (7) is then ill-conditioned. We also tested using two other initial conditions namely $\mathrm{L}(0)=40000$ and 

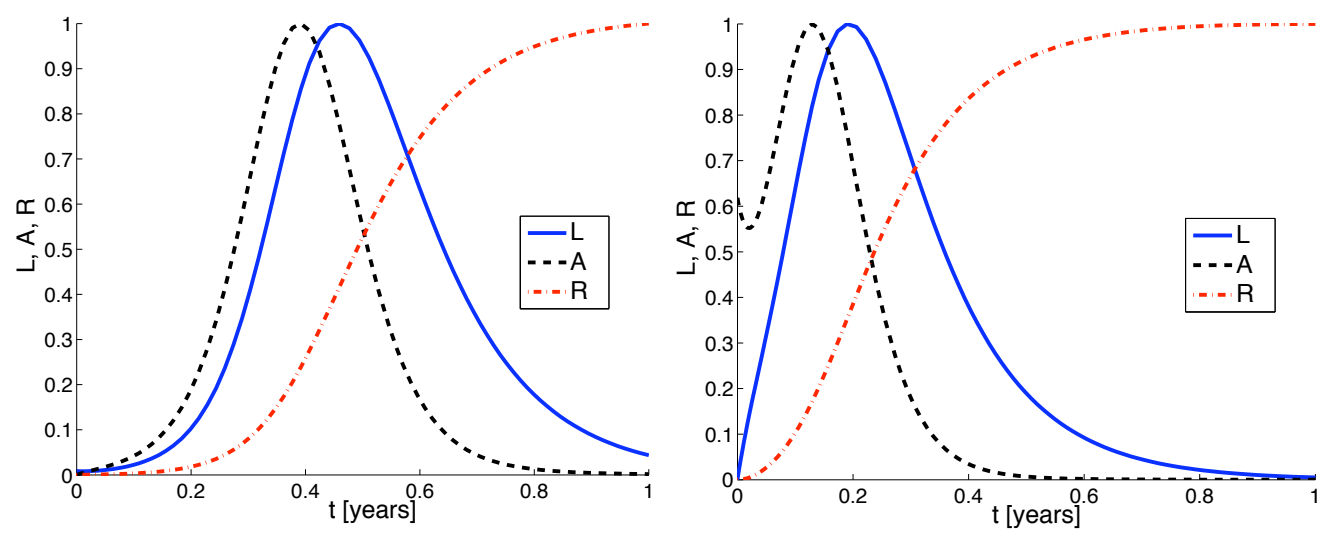

Figure 1: Scaled solutions $L(t), A(t)$ and $R(t)$ to Equations (1)-(3) using Equations (8)-(10). Initial conditions are all zero except in the left panel $\mathrm{L}(0)=40000$ while in the right panel $\mathrm{A}(0)=4000$.

$\mathrm{L}(0)=400$ and found that poor results were obtained, because Equation (7) is then ill-conditioned. That is, while two experiments are needed to be held under similar enough conditions for them to be testing the same thing, the experiments must be different enough that the equations are not illconditioned. This is a challenge to experimentalists. We note that if $\rho$ is estimated by other means then $X_{1}(R)$ is easily found using one experiment and Equation (1).

Typical results from 1974 experiments [10, Figures 1 and 2] on Ostertagia circumcincta and Trichostrongylus are shown in Figures 3 and 4 along with approximate functional forms for $\mathrm{L}$

$$
L(t)=l_{0}+\frac{l_{\max } b^{2}}{b^{2}+\left(t-t_{l}\right)^{2}}
$$

and similarly for $A(t)$. While these approximations do not capture all of the details in the data, they do represent reasonable heuristic fits, capturing the essential elements of both sets of data. The derivatives $d A / d t$ and $d L / d t$ are taken from the analytic derivatives of Equation (11). 


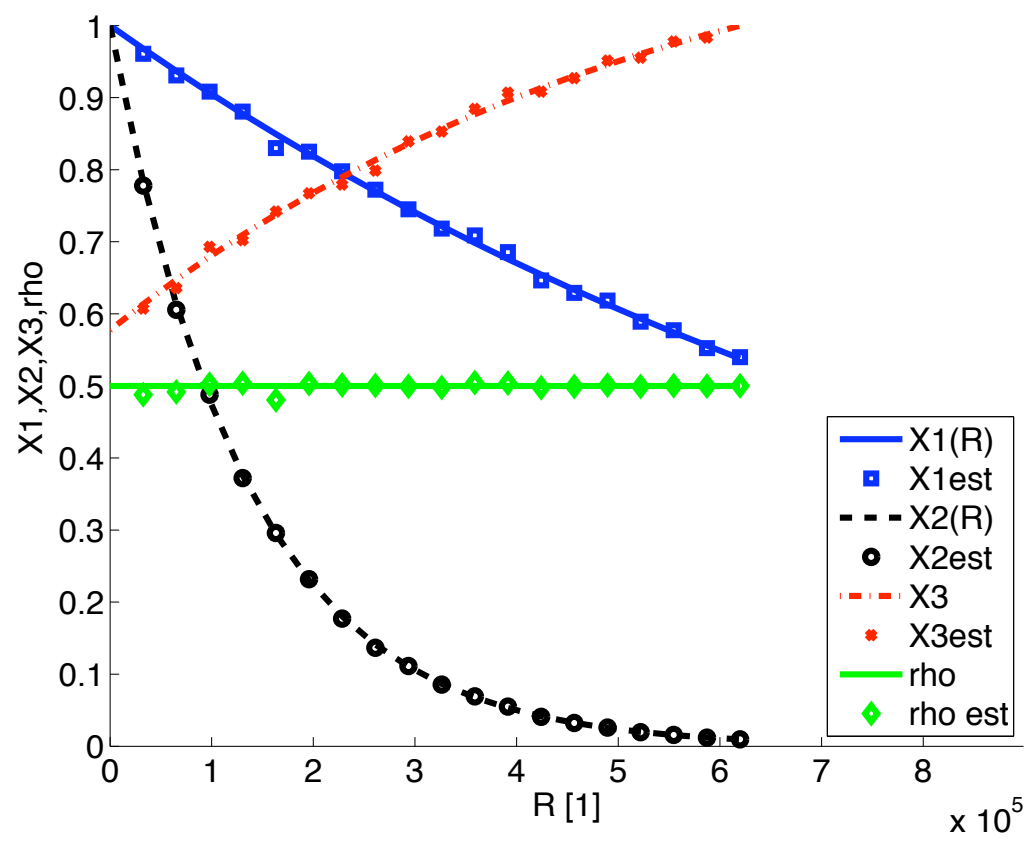

FiguRE 2: Regenerating $X_{1}(R), X_{2}(R)$ and $X_{3}(R)$ at 20 points, scaled respectively by $13797,0.12775$ and 21.7973 . The original assumed forms are shown as the continuous curves. The value of $\rho=7$ is also shown scaled by 14 for convenience. 


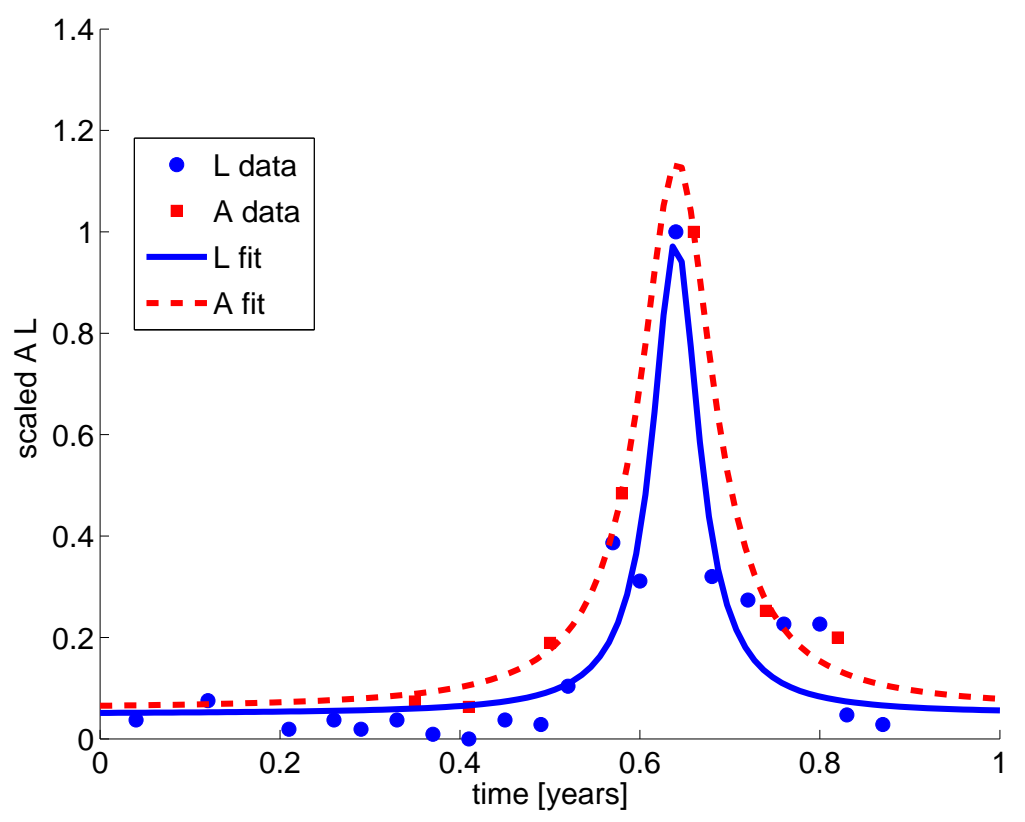

FiguRE 3: The population of Ostertagia circumcincta larvae (circles) and adults (squares) taken from a 1974 study [10]. The curves are fits to the data of arbitrary functions (11): $l_{0}=58.98, l_{\max }=1124, b=0.0313$, $\mathrm{t}_{1}=0.640, \mathrm{a}_{0}=656, \mathrm{a}_{\max }=12040, \mathrm{~b}=0.0492$, and $\mathrm{t}_{\mathrm{a}}=0.641$. 


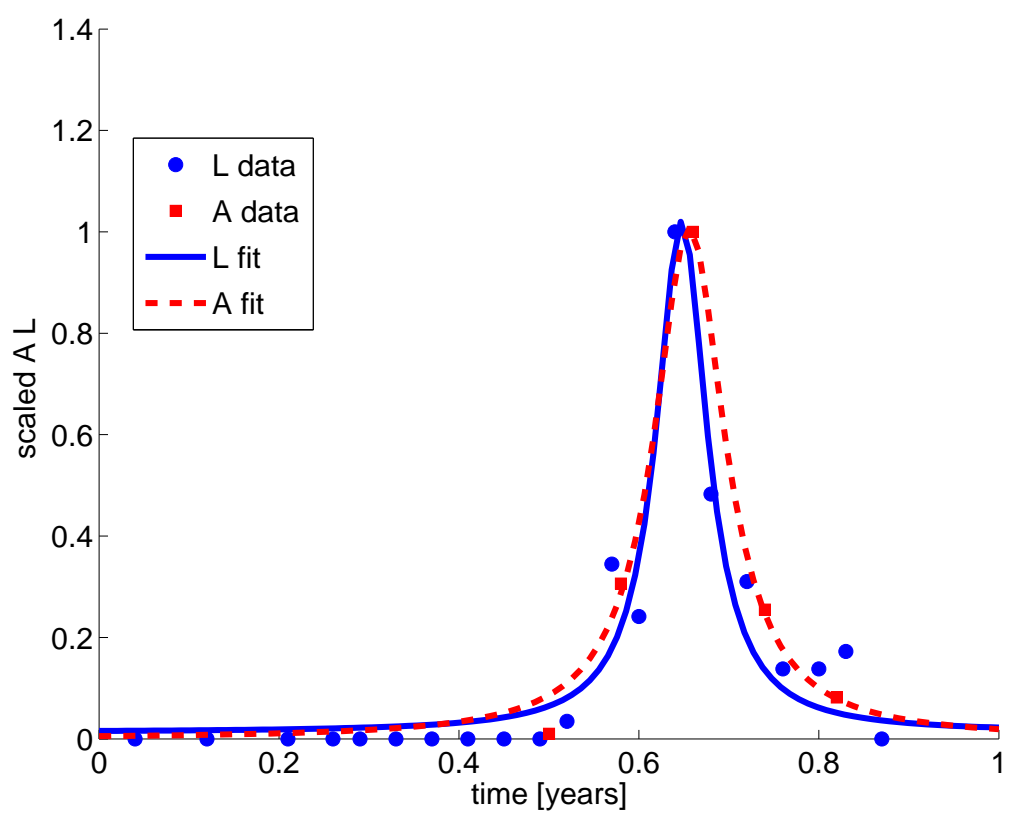

FiguRE 4: The population of Trichostrongylus larvae (circles) and adults (squares) taken from a 1974 study [10]. The curves are fits to the data of arbitrary functions (11): $l_{0}=57.7, l_{\max }=1125, b=0.032, t_{1}=0.638$, $a_{0}=738, a_{\max }=12390, d=0.045$, and $t_{a}=0.683$. 


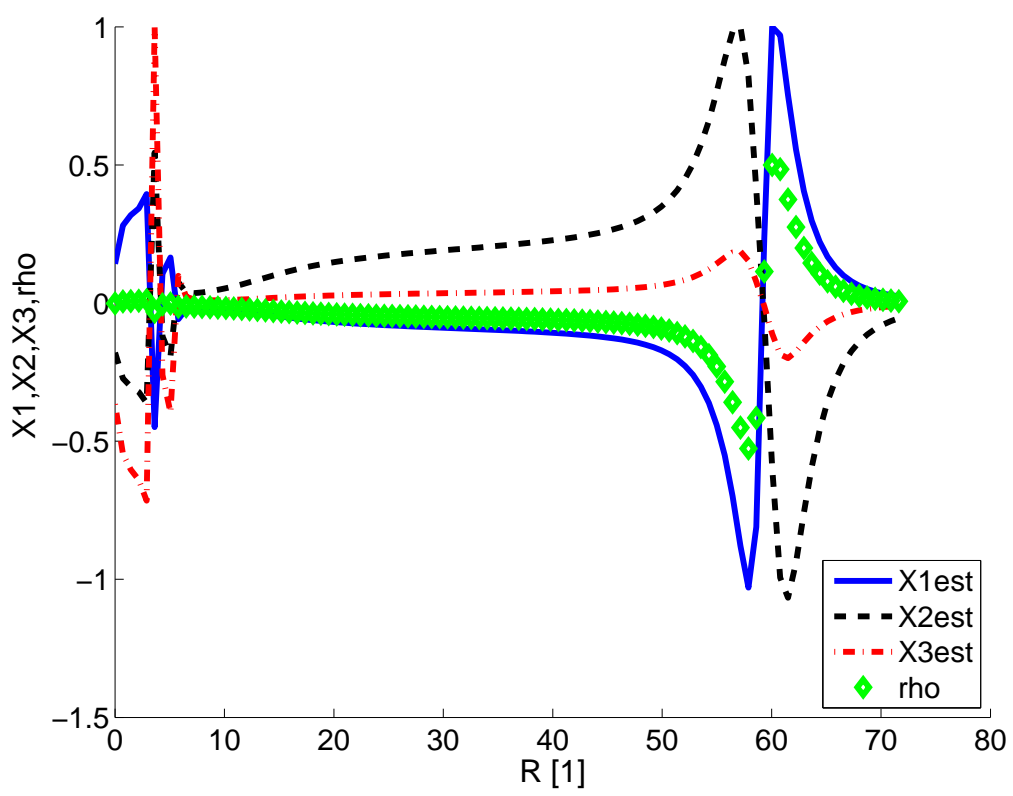

Figure 5: Calculations of $X_{i}(R)$ using Equation (7) based on the parametric fits shown in Figure 3 based on experimental data [10].

When using these data, along with similar experimental data [10] we obtained non-physical results for $X_{1}(R), X_{2}(R), X_{3}(R)$ and $\rho$. That is, while our method gave mathematical results for $X_{i}$ they were not physically meaningful, becoming negative and varying considerably with R. Figure 5 shows typical results, with $\rho, X_{1}, X_{2}$ and $X_{3}$ scaled by 31, 0.2606, 998 and 16 respectively. The wild oscillations for small $R$ are due to ill-conditioning when experimental data are poor at small time. The failure to find $X_{i}(R)$ for this set of experiments has several rationalisations: the experiments may be too similar causing ill-conditioning; as the experiments were run in consecutive years, the physical conditions might not have been similar enough for the experiments to be compared; or the model presented here, Equations (1)-(3), is not an accurate reflection of the parasite-host interaction. 
These results indicate the difficulty of designing experiments which allow the functions $X_{i}(R)$ to be found. To avoid ill-conditioning it is required that $A(0)=0$ and $L(0)>0$ in one experiment, and $A(0)>0$ and $L(0)=0$ in a second. Initially, such experiments require parasite-naive animals and parasite-free pasture. Half of the animals should be infected with larvae and the remainder maintained free of parasites, and all the animals housed until eggs appear in the faeces of the infected animals indicating the presence of adult parasites in those animals. One half of the parasite-free pasture should be infected with parasites (ideally using larvae from the same source as those used to infect the animals) and the other half should be maintained free of parasites. The infected animals should then be grazed on the parasitefree pasture $(A(0)>0, L(0)=0)$ and the parasite-naive animals should be grazed on the infected pasture $(A(0)=0, L(0)>0)$. The populations of adults and larvae should then be monitored for each experiment and our solution method used to find $X_{i}(R)$. If, in this circumstance, the functions cannot be found, then there is cause to reconsider the viability of this particular model. Hence the next section describes an alternative model which mimics some of the behaviour of parasite host interactions.

\section{An alternative model}

We demonstrate here an alternative model of the parasite lifecycle. We hypothesize that the larval density $L(t)$ and adult density $A(t)$ are governed by two sets of delay differential equations. When the adult density is smaller than some critical adult density, $A^{*}$, then

$$
\begin{aligned}
\frac{d L}{d t} & =-X_{0} L+X_{1} A\left(t-\tau_{1}\right), \\
\frac{d A}{d t} & =X_{2} L\left(t-\tau_{2}\right)-X_{3} A,
\end{aligned}
$$

where $X_{0}=\beta+\rho, X_{1}, \ldots, X_{3}$ represent the same coefficients as in earlier equations, $\tau_{1}$ and $\tau_{2}$ represent the delay in larvae becoming egg-producing 
adults, and for eggs to become develop into larvae. These delays are of the order of 30 days and 10 days, respectively [3] which although relatively small on the order of a year, prove quite crucial on the time scales of the parasite peak. The coefficients $X_{i}$ are considered constant during this growth phase (when $\left.A<A^{*}\right)$. However, when there are sufficient adult parasites $\left(A \geq A^{*}\right)$, host resistance eliminates parasite egg production and the development of larvae to adults. Hence for $A>A^{*}$ the system moves towards

$$
\begin{aligned}
& \frac{d L}{d t}=-Y_{0} L, \\
& \frac{d A}{d t}=-Y_{3} A,
\end{aligned}
$$

for some $Y_{0} \approx X_{0}$ and $Y_{3}$. This second set of equations simply demonstrates the exponential decay of larval and adult density apparent after the peak in the data. This model reduces to the earlier model if $\tau_{1}$ and $\tau_{2}$ are taken as zero and the dependence on resistance modified so that $R \propto A$. This step function approach without time delays has been used by Roberts and Heesterbeek (1998) [7] although they applied the step function to a normalised version of $Q(R)=X_{1} X_{2} / X_{3}(\rho+\beta)$ and using a transformed set of equations.

Due to the time delay, full analysis of this model is difficult, and beyond the scope of this current article. However, Figure 6 illustrates a typical result us$\operatorname{ing} X_{0}=17, X_{1}=4.9, X_{2}=660, X_{3}=4.32, Y_{0}=17, Y_{3}=14, \tau_{1}=36 / 365$ and $\tau_{2}=50 / 365$, expressed in years. Initial values were $L(0)=18$ and $A(0)=0$. Results were scaled with respect to the maximum density of larvae (1205) and adults (11333). Finding the optimal values of the coefficients $X_{1}, \ldots, \tau_{2}$ is difficult due to the nonlinearities associated with the time delays. However, in obtaining the results plotted in Figure 6, it was evident that a significant time delay is necessary to account for the initially small values of parasite numbers followed by a rapid rise, something not found when using the resistance model, Equations (1)-(3). In addition, the sharp peak exhibited in the experimental data is not reproduced well by this earlier resistance model, while this alternative time delay model with the abrupt 


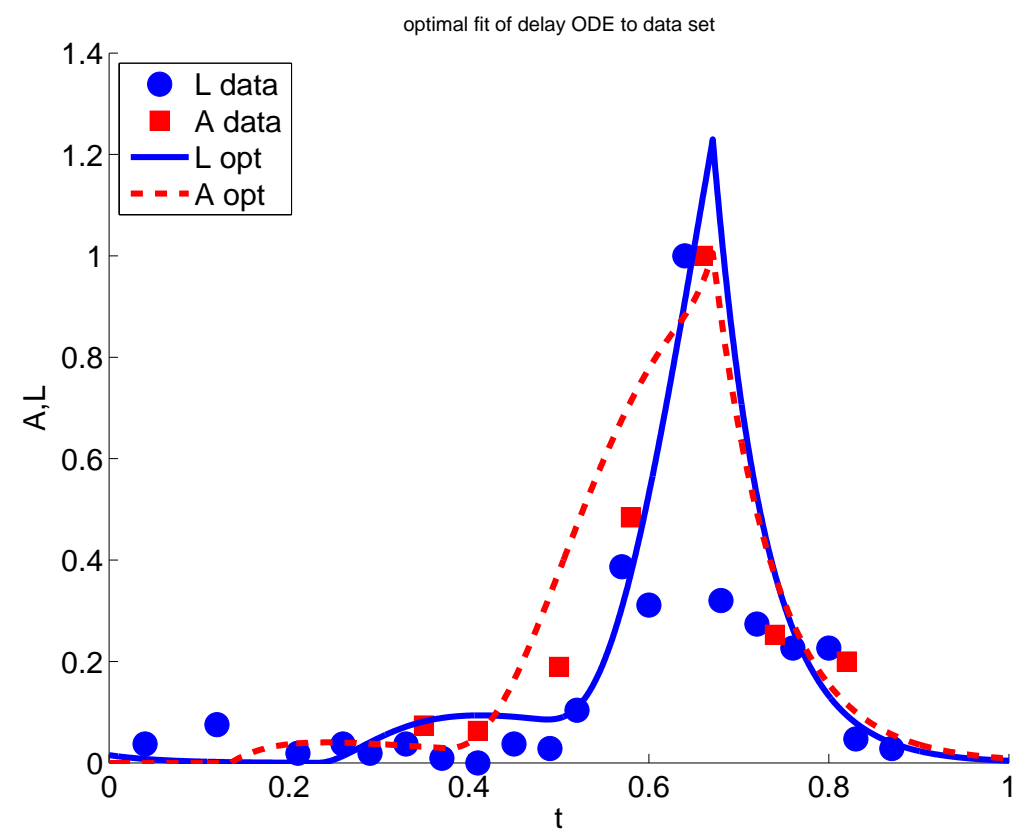

Figure 6: Adult and larval densities calculated using Equations (12)-(15) compared with experimental data [10]. 
behaviour change at $A=A^{*}$ does model this peak and the resultant exponential decay in the data. Interestingly, both $L$ and $A$ increase in two steps, which is a common feature of much of the experimental data $[2,9]$.

All models of this type, including the original model, are simply mathematical expressions based on reasonable physical assumptions which hopefully produce results to accurately replicate experimental data. The model then gives some insight into the underlying mechanisms of parasite-host behaviour and hence some predictive capabilities. Our alternative model is an extension of the earlier model making some additional assumptions. It appears from our preliminary study that this alternative model replicates key features of the observed data better than the original model. This indicates to researchers the potential importance of time delays and step function coefficients in future models. We hope that further experiments provides data allowing verification and modification of the model, hence enabling researchers to gain a better understanding of the fundamental dynamics of parasite growth.

\section{Conclusion}

This work illustrates appropriate methods for finding the functions $X_{i}(R)$ in Equations (1)-(3) given two sets of experimental data. The method is tested using numerical experiments and found to be accurate. However, when tested on experimental data the method yields physically unrealistic estimates of the functions $X_{i}(R)$. Hence we believe the initial resistance model may not accurately reflect reality. We propose an alternative model which includes time delay terms, and initial results look promising. Further work is necessary to find a method of accurately finding the coefficients $X_{1}, \ldots, \tau_{2}$ in the time delay model from experimental data. 


\section{References}

[1] Anderson, R. M. and May, R. M. Herd immunity to helminth infection and implications for parasite control. Nature 315, 493-496, 1985. doi:10.1038/315493a0 C698

[2] Gordon, H. M. The epidemiology of parasitic diseases, with special reference to studies with nematode parasites of sheep. Australian Veterinary Journal 24, 17-45, 1948. doi:10.1111/j.1751-0813.1948.tb01569.x C710

[3] Hansen, J. and Perry, B. The epidemiology, diagnosis and control of helminth parasites of ruminants. International Laboratory for Research on Animal Diseases, 1994. C708

[4] Kao, R. R., Leathwick, D. M., Roberts, M. G. and Sutherland, I. A. Nematode parasites of sheep: a survey of epidemiological parameters and their application in a simple model. Parasitology 121, 85-103, 2000. http://journals. cambridge.org/action/displayIssue?jid= PAR\&volumeId=121\&issueId=01\&iid=53786 C698, C699

[5] Louie, K., Vlassoff, A. and Mackay, A. Nematode parasites of sheep: extension of a simple model to include host variability. Parasitology 130, 437-446, 2005. doi:10.1017/S003118200400678X C698, C699, C700

[6] Roberts, M. G. and Grenfell, B. T. The population dynamics of nematode infections of ruminants: periodic perturbations as a model for management. IMA Journal of Mathematics Applied in Medicine and Biology 8, 83-93, 1991. doi:10.1093/imammb/8.2.83 C697, C698, C699, C700, C701

[7] Roberts. M. G. and Heesterbeek, J. A. P. A simple parasite model with complicated dynamics. J. Math. Biol., 37: 272-290, 1998. doi:10.1007/s002850050129 C708 
[8] Sargison, N. D, Wilson, D. J, Bartley, D. J, Penny, C. D and Jackson, F. Haemonchosis and teladosagiosis in a Scottish sheep flock putatively associated with the overwintering of hypobiotic fourth stage larvae, Veterinary Parasitology 147, 326-331, 2007. doi:10.1016/j.vetpar.2007.04.011 C701

[9] Vlassoff, A., Leathwick, D. M. and Heath, A. C. G. The epidemiology of nematode infections of sheep. New Zealand Veterinary Journal 49, 213-221, 2001. http://www. sciquest.org.nz/default.asp?pageid= $69 \& \mathrm{pub}=2 \& \mathrm{vol}=49 \& \mathrm{iss}=6 \mathrm{C} 710$

[10] Waller, P. J., Dobson, R. J., Donald, A. D. and Thomas, R. J. Populations of strongylid nematode infective stages in sheep pastures: comparison between direct pasture sampling and tracer lambs as estimators of larval abundance. International Journal of Parasitology 11, 359-367, 1981. doi:10.1016/0020-7519(81)90006-0 C702, C704, C705, C706, C709

\section{Author addresses}

1. Steven I. Barry, University of New South Wales @ ADFA, Canberra, Australia. mailto:s.barry@adfa.edu.au

2. Simon Brown, School of Human Life Sciences, University Tasmania, Launceston, Australia.

mailto:Simon.Brown@utas. edu . au 